\title{
ON CONJUGATE POWERS IN EIGHTH-GROUPS
}

\author{
BY SEYMOUR LIPSCHUTZ
}

Communicated by Everett Pitcher, April 21, 1971

Let $G$ be a finitely presented group with generating elements $a_{1}, \cdots, a_{\lambda}$ and defining relations $R_{1}=1, \cdots, R_{\mu}=1$. We assume without loss of generality that the relators $R_{i}$ form a symmetric set, i.e. that the $R_{i}$ are cyclically reduced and are closed under the operations of taking inverses and cyclic transforms. We call $G$ an eighth-group if it satisfies the following condition:

(*) If $R_{i} \cong X Y$ and $R_{j} \cong X Z$ are distinct relators, then the length of the common initial segment $X$ is less than $1 / 8$ the length of either relator.

A classical example of such a group is the fundamental group $G_{k}$ of an orientable closed 2-manifold of genus $k>2$; it has the presentation

$$
G_{k}=\operatorname{gp}\left(a_{1}, b_{1}, \cdots, a_{k}, b_{k} ; a_{1} b_{1} a_{1}^{-1} b_{1}^{-1} \cdots a_{k} b_{k} a_{k}^{-1} b_{k}^{-1}=1\right) .
$$

More generally, the Fuchsian groups $F\left(p ; n_{1}, \cdots, n_{d} ; m\right)$, see Greenberg [3], are eighth-groups if $4 p+d+m, n_{1}, \cdots, n_{d}>8$.

The class of eighth-groups were first considered by Greendlinger who solved the word problem [4] and the conjugacy problem [5] for them. Similar "small cancellation" groups have been studied by Tartakovskii [8], Britton [1], Lyndon [6] and Schupp [7], among others.

We now state our main result.

TheOREM. Suppose $W$ is an element of infinite order in an eighthgroup G. If $|m| \neq|n|$ then $W^{m}$ and $W^{n}$ are in different conjugacy classes. In particular, $W, W^{2}, W^{3}, \cdots$ are in different conjugacy classes.

This theorem has already been known to hold for the above fundamental groups $G_{k}$ and for the Fuchsian groups; but all the proofs have been topological. Our theorem holds for a much wider class of groups and, moreover, the proof is purely algebraic.

The author conjectures that the theorem also holds for the small concellation groups in general.

AMS 1969 subject classifications. Primary 2010; Secondary 2070.

Key words and phrases. Small cancellation groups, Greendlinger groups, conjugacy classes. 
We also note that Gowdy [2] has classified those elements in eighth-groups which are conjugate to their inverses, and showed that no such elements exist if the group is torsion-free.

\section{BIBLIOGRAPHY}

1. J. L. Britton, Solution of the word problem for certain types of groups. I, Proc. Glasgow Math. Assoc. 3 (1956), 45-54. MR $20 \# 3205$.

2. S. Gowdy, On Greendlinger eighth-groups, Ph.D. Thesis, Temple University, Philadelphia, Pa., 1971.

3. L. Greenberg, Discrete groups of motions, Canad. J. Math. 12 (1960), 415-426. MR $22 \# 5932$.

4. M. Greendlinger, Dehn's algorithm for the word problem, Comm. Pure Appl. Math. 13 (1960), 67-83. MR 23 \#A1693.

5. - On Dehn's algorithm for the conjugacy and word problems, with applications, Comm. Pure Appl. Math. 13 (1960), 641-677. MR 23 \#A2327.

6. R. C. Lyndon, On Dehn's algorithm, Math. Ann. 166 (1966), 208-228. MR 35 \#5499.

7. P. E. Schupp, On Dehn's algorithm and the conjugacy problem, Math. Ann. 178 (1968), 119-130. MR 38 \#5901.

8. V. A. TartakovskiY, Solution of the word problem for groups with a k-reduced basis for $k>6$, Izv. Akad. Nauk SSSR Ser. Mat. 13 (1949), 483-494; English transl., Amer. Math. Soc. Transl. (1) 1 (1962), 211-227. MR 11, 493.

Temple University, Philadelphia, Pennsylvania 19122 